\title{
An Initial Survey on Insect Associated Mites of South Bengal
}

\author{
Ananya Das ${ }^{1}$, Salil K. Gupta ${ }^{2}$ \\ ${ }^{1,2}$ (PG Department of Zoology, Vidyasagar College, Kolkata, India)
}

\begin{abstract}
A preliminary study related to mites associated in the insects is given in this article. Ten species of mites under nine families collected from six orders of insects from South Bengal are reported here giving collection data and biological information.
\end{abstract}

Keywords - Coleoptera, Diptera, Hemiptera, Hymenoptera, Insects, Lepidoptera, Mites, Odonata, South Bengal

\section{INTRODUCTION}

Members of various orders of insects are often found infested by mites of different groups and the association between them may be of temporary nature for passive transport or may be commensalistic, parasitic or may be of predatory nature (Hunter and Rossario, 1988). The parasitic /predatory behaviour of mites, may be exploited in biological control of insect pests and hence, insect associated mites have received importance and studies have been initiated to explore the mites associated with insects, especially of agricultural importance in order to find out the nature of association between them. However, most of the published works available from India are from South India. Therefore, the present study was undertaken to procure some preliminary idea on the insect associated mites of West Bengal.

\section{MATERIALS AND METHODS}

Insects were collected from different agricultural and horticultural fields located in South 24 Paraganas, district of West Bengal with the help of a collecting net and light trap. The insect specimens thus collected were thoroughly examined under a stereo-binocular microscope for collecting the mites associated with them. The insect groups collected and examined for mites were members of the orders Lepidoptera, Hemiptera, Diptera, Coleoptera, Hymenoptera and Odonata. Different parts of the insect body like the underside of proboscis and elytra, wing base, legs, area between head and thorax, antennae, etc. were examined for collection of mites. The collected mite specimens were temporarily mounted in lactic acid for microscopic observation. Permanent slides were prepared in Hoyer's medium. The slide mounted specimens were examined and identified under a research microscope, following appropriate identification keys and literature. The identification of host insects was made mostly by the experts of the Zoological Survey of India, Kolkata.

\section{RESULTS AND DISCUSSIONS}

The examination of the collected specimens of mites revealed the occurrence of 10 species under 10 genera, 9 families and 3 orders from members of 6 orders of insects Table-1.

\begin{tabular}{|c|c|c|c|c|c|}
\hline $\begin{array}{l}\text { Sl. } \\
\text { No. }\end{array}$ & Order / Family & Species & Host & Location & Remarks \\
\hline 1 & $\begin{array}{c}\text { ORDER I } \\
\text { MESOSTIGMATA } \\
\text { Family 1: } \\
\text { Eviphidae }\end{array}$ & $\begin{array}{l}\text { Alliphis sp. } \\
\text { Alliphis sp. } \\
\text { Alliphis sp. }\end{array}$ & $\begin{array}{c}\text { Lepidoptera } \\
\text { (Junonia } \text { sp.) } \\
\text { Lepidoptera } \\
\text { (Papilio demoleus) } \\
\text { Linn. } \\
\text { Lepidoptera } \\
\text { (Agrotis } \text { sp.) }\end{array}$ & $\begin{array}{c}\text { Baruipur } \\
\text { Narendrapur } \\
\text { Science City } \\
\text { Area }\end{array}$ & $\begin{array}{l}\text { all under } \\
\text { proboscis, } \\
\text { below } \\
\text { wing base, } \\
\text { leg }\end{array}$ \\
\hline 2 & $\begin{array}{l}\text { Family 2: } \\
\text { Phytoseiidae }\end{array}$ & $\begin{array}{c}\underline{\text { Amblyseius }} \\
\text { largoensis(Muma) }\end{array}$ & $\begin{array}{c}\text { Hemiptera } \\
\text { Leptocorisa } \\
\text { acuta(Thunb.) }\end{array}$ & Chinsura & $\begin{array}{c}\text { between } \\
\text { head and } \\
\text { thorax, below } \\
\text { elytra }\end{array}$ \\
\hline 3 & $\begin{array}{c}\text { Family 3: } \\
\text { Otopheido-menidae }\end{array}$ & $\frac{\text { Hemipteroseius }}{\text { indicus (Krantz \& Khot) }}$ & $\begin{array}{c}\text { Hemiptera } \\
\text { Dysdercus } \\
\text { koenigii Fabr. }\end{array}$ & $\begin{array}{l}\text { Narendrapur } \\
\text { Kalikapur }\end{array}$ & $\begin{array}{l}\text { ventrally on } \\
\text { thorax }\end{array}$ \\
\hline 4 & $\begin{array}{c}\text { Family4: } \\
\text { Macrochelidae }\end{array}$ & $\frac{\frac{\text { Macrocheles }}{\text { muscaedomesticae }}}{\frac{(\text { Scopoli })}{\#}}$ & $\begin{array}{c}\text { Diptera } \\
\text { Musca domestica } \\
\text { Linn. } \\
,,\end{array}$ & $\begin{array}{l}\text { Baruipur } \\
\text { Baruipur }\end{array}$ & $\begin{array}{l}\text { below } \\
\text { antennae, } \\
\text { wing base }\end{array}$ \\
\hline
\end{tabular}




\section{A Preliminary Report on Insect Associated Mites of South Bengal}

\begin{tabular}{|c|c|c|c|c|c|}
\hline 5 & $\begin{array}{l}\text { Family 5: } \\
\text { Ascidae }\end{array}$ & $\begin{array}{l}\text { Blattisocius keegani } \\
\text { (Fox) }\end{array}$ & $\begin{array}{l}\text { Lepidoptera } \\
\text { (Danais sp.) }\end{array}$ & Kalikapur & $\begin{array}{c}\text { under } \\
\text { proboscis, } \\
\text { below } \\
\text { wing base, } \\
\text { leg }\end{array}$ \\
\hline 6 & $\begin{array}{l}\text { Family 6: } \\
\text { Eviphidae }\end{array}$ & Eviphis sp. & $\begin{array}{c}\text { Coleoptera } \\
\text { (Henosepilachna } \\
\text { vigintioctopunctata) } \\
\text { Fabr. }\end{array}$ & $\begin{array}{c}\text { Science City } \\
\text { area }\end{array}$ & $\begin{array}{l}\text { below } \\
\text { elytra }\end{array}$ \\
\hline 7 & $\begin{array}{c}\text { ORDER II } \\
\text { ASTIGMATA } \\
\text { Family 1: } \\
\text { Acaridae } \\
\end{array}$ & $\begin{array}{c}\frac{\text { Rhizoglyphus echinopus }}{\text { (F. and R) }} \\
\text { " }\end{array}$ & $\begin{array}{c}\text { Coleoptera } \\
\text { (Aspidomorpha sp.) }\end{array}$ & $\begin{array}{c}\text { Baruipur } \\
\text { Narendrapur }\end{array}$ & $\begin{array}{c}\text { on ventral } \\
\text { surface, } \\
\text { area between } \\
\text { head \& thorax }\end{array}$ \\
\hline 8 & $\begin{array}{l}\text { Family 2: } \\
\text { Anoetidae }\end{array}$ & $\begin{array}{c}\text { Sennertia sp. } \\
,\end{array}$ & $\begin{array}{l}\text { Hymenoptera } \\
\text { Xylocopa sp. }\end{array}$ & $\begin{array}{l}\text { Narendrapur } \\
\text { Baruipur }\end{array}$ & $\begin{array}{l}\text { on ventral } \\
\text { surface }\end{array}$ \\
\hline 9 & $\begin{array}{l}\text { Family 3: } \\
\text { Acaridae }\end{array}$ & $\begin{array}{c}\text { Caloglyphus berlesei } \\
\text { (Michael) }\end{array}$ & $\begin{array}{c}\text { Coleoptera Coccinella } \\
\text { sp. }\end{array}$ & Narendrapur & $\begin{array}{c}\text { on ventral } \\
\text { surface, } \\
\text { area between } \\
\text { head \& thorax }\end{array}$ \\
\hline 10 & $\begin{array}{c}\text { ORDER III } \\
\text { PROSTIGMATA } \\
\text { Family: } \\
\text { Arrenuridae }\end{array}$ & Arrenurus sp. & Odonata & $\begin{array}{l}\text { Science City } \\
\text { area }\end{array}$ & $\begin{array}{c}\text { below } \\
\text { antennae, } \\
\text { area between } \\
\text { head \& thorax }\end{array}$ \\
\hline
\end{tabular}

Table-1: Details of species collected from insects of South Bengal

As presented in Table-1, the mesostigmatid mites collected during the study were Alliphis sp., Amblyseius largoensis (Muma), Hemipteroseius indicus, Krantz \& Khot Macrocheles muscaedomesticae,(Scopoli), Blattisocius keegani, Fox and Eviphis sp. of these, Alliphis sp. and Blattisocius keeagni collected from Lepidoptera, Hemipteroseius indicus from red cotton bug and Macrocheles muscaedomisticae from house fly - were the most common species. Similar findings were reported by Ramaraju (2009) also from Tamil Nadu. However, the Alliphis sp. collected during the present study appears to be different from Alliphis trichiensis Ramaraju and Mohanasundaram and also from all the other known species. This clearly indicates that the present species of Alliphis may probably represent a new taxon. Ramaraju (2009) reported 17 species under 17 genera and the present study reports only 6 species. The recovery of A.largoensis during the present study appears to be a case of accidental occurrence as it is a leaf inhabiting predatory mite. The association of other mesostigmatid mites was phoretic.

The present report includes 3 species of astigmatid mites, of which Sennertia sp. appears to be a new one. The other 2 species of this order were truly phoretic. Ramaraju et al. Ramaraju (2009), and Ramaraju \& Mohanasundaram $(1998,1999)$ reported 7 species of this order from Tamil Nadu, associated with insects.

In the present study, only one species of Protsigmata i.e. Arrenurus sp. could be collected while Ramaraju (2009) reported 9 species of Prostigmata under 9 genera. The present work though represents a preliminary study; it enabled to understand the rich diversity of mites associated with insects. More in-depth studies are warranted to disclose the faunal diversity of insect associated mites of North India.

\section{Acknowledgements}

The authors are thankful to Dr G.C. Sadhukhan, P.G. Dept of Zoology, Vidyasagar College, Kolkata for providing infrastructure facilities. Sincere thanks are also due to the Secretary, Ramakrishna Mission Ashrama, Narendrapur for permitting the authors to conduct this study (collection of insects etc) in the campus of the Ashrama.

\section{REFERENCES}

[1] Hunter, P.E. and Rossario, R.M.T. (1998), Associations of Mesostigmata with other arthropods, Ann.Rev.Ent.33.319.

[2] Ramaraju, K. \& Mohanasundaram, M. (1998), New phoretic mites (Acari; Chaetodactylidae) on carpenter bees from Tamil Nadu, India, Internat. J. Acarol. 27 (2), 107-112

[3] Ramaraju, K. \& Mohanasundaram, M. (1999), A new hypopus of Calvolia longireticulatae sp.nov. (Acari; Winterschmidtiidae) from Tamil Nadu, India J.Ent. Res. 23(1), 47-49

[4] Ramaraju, K. 2009 Biodiversity of mites associated with insects in Western Ghats, GOI-MOEF Project. Annual progress report 2008-2009, Department of Agricultural Entomology, Center for plant protection studies, Tamil Nadu Agricultural University, Coimbatore 641003, 53 